The seroincidence was high (55\%) even when the group immunity had already been partially established (42\%) and the Ae. aegypti infestation rates were relatively low $(<3 \%)$. Contrary to the ecological analysis, at the individual level, substantial heterogeneity in dengue exposure was observed.

Conclusion The observation that the dengue virus in our environment does not respect social spaces strengthens the principle that vector control measures must always be universally applied in each territory. On the other hand, the identification of specific risk factors in the domestic domain may indicate a need for other evidencebased interventions which can help to eliminate the disease from cities such as Salvador.

\section{P2-546 AGE AT DIAGNOSIS AFFECTS ADHERENCE TO NATIONAL BREAST CANCER GUIDELINES REGARDING LOCAL THERAPY}

doi:10.1136/jech.2011.142976m.73

W van de Water, E Bastiaannet, ${ }^{*}$ A J M de Craen, C J H van de Velde, G J Liefers. Leiden University Medical Centre, Leiden, The Netherlands

Introduction In the Netherlands, breast cancer patients are treated according to national guidelines. Despite comprising a large proportion, elderly breast cancer patients are underrepresented in clinical trials. Therefore it is questionable whether guideline recommendations can be extrapolated from a young to a heterogeneous elderly population. Aim of this study was to assess age specific guideline adherence.

Methods Data were extracted from the Dutch Cancer Registration. Overall, 83982 patients without distant metastases, diagnosed between 2002 and 2008 were included. Adherence was assessed for breast surgery (BS), type of surgery (TS), axillary surgery (AS) and radiotherapy $(\mathrm{RT})$. Analyses were stratified by age $(<65,65-74$ and $\geq 75$ years)

Results Adherence decreased with increasing age (BS <65, 99\%; $65-74,98 \%$; $\geq 75,75 \%$; $<<0.001$. TS $69 \%$; 66\%; 42\% respectively, $\mathrm{p}<0.001$. AS 96\%; 94\%; 68\% respectively, $\mathrm{p}<0.001$. RT 91\%; 93\%; $83 \%$ respectively, $\mathrm{p}<0.001)$. BS and AS non adherence were explained by under treatment. For TS and RT, young patients were non adherent because of over- and under treatment. In the elderly, under treatment was the main reason for non adherence (under treatment TS 84\%; 91\%; 97\% respectively, p $<0.001$. RT 34\%; $50 \% ; 80 \%$ respectively, $\mathrm{p}<0.001)$. Over time adherence to breast surgery declined for elderly patients $(p<0.001)$. For other local therapies, the gap between young and elderly patients remained similar.

Conclusion These results emphasise the different approach and treatment of elderly breast cancer patients. Further age specific studies in breast cancer patients are necessary to investigate whether adherence to current guidelines results in optimal outcomes for elderly patients.

\section{P2-547 TILE: MUSCULOSKELETAL DISORDERS AND ASSOCIATED FACTORS AMONG NURSES IN SRI LANKA}

doi:10.1136/jech.2011.142976m.74

${ }^{1} \mathrm{~S}$ Warnakulasuriya, ${ }^{*} \mathrm{R}$ Peiris Jone, ${ }^{2} \mathrm{~A}$ R Wickramasinghe, ${ }^{3} \mathrm{~N}$ Sathiakumar. ${ }^{1}$ Faculty of Medical Sciences, University of Sri Jayewardenapura, Colombo, Sri Lanka; ${ }^{2}$ Faculty of Medicin, University of Kelaniya, Ragama, Sri Lanka; ${ }^{3}$ School of Public Health, University of Alabama, Berminham, USA

Introduction Work related musculoskeletal disorders (WMSDs) have significant impacts in labour intensive occupational groups. Lifting, moving, transferring and repositioning of patients and long hours spent standing during work makes nurses vulnerable to WMSDs.

Objective To determine the prevalence and risk factors associated with WMSDs among nurses.

Methods We randomly selected 237 nurses attached to National hospital of Sri Lanka and Colombo South Teaching hospitals, and interviewed them using a pre-tested questionnaire ascertaining WMSDs in the past year. Descriptive, $\chi^{2}$ tests and multivariate logistic regression analyses were performed.

Results Results indicated that $21 \%$ of nurses had at least one WMSD. Back pain was the most common WMSD occurring in $44 \%$ of nurses. The prevalence of other WMSDs included pain in the knee $(30 \%)$, neck $(19 \%)$, shoulder $(16 \%)$ wrist and arm $(13 \%)$ and elbow (4\%). Lower back pain was significantly associated with time pressure $(\mathrm{OR}=2.14,95 \% \mathrm{CL} 1.17$ to 3.88$)$, job dissatisfaction $(\mathrm{OR}=6.84,95 \%$ CL 1.46 to 31.94$)$, lifting more than $25 \mathrm{~kg}$ during a normal duty shift ( $\mathrm{OR}=2.16$, CI 1.26 to 3.7), and unhappiness during the past month $(\mathrm{OR}=2.74,95 \% \mathrm{CI} 1.06$ to 7.08$)$. Shoulder pain was significantly associated with job dissatisfaction $(\mathrm{OR}=4.15,95 \%$ CI 1.24 to 13.87$)$ and unhappiness ( $\mathrm{OR}=2.98,95 \%$ CI 1.11 to 7.98 )

Conclusion The present study shows that nurses have a high prevalence of MSDs. The predominant ergonomic problem is lower back pain, followed by pain in the knee, neck and shoulder areas. Interventional studies are needed to address WMSDs among nurses, particularly for lower back pain.

\section{P2-548 GERIATRIC MENTAL HEALTH AND RELIGION: RESULTS FROM THE KRAKOW STUDY ON THE RELATIONSHIP BETWEEN RELIGIOUS INVOLVEMENT AND HEALTH IN OLDER AGE}

doi:10.1136/jech.2011.142976m.75

B Wozniak, ${ }^{*}$ K Zawisza, B Tobiasz-Adamczyk. Jagiellonian University Medical College, Krakow, Poland

Background Taking into account the prevalence of religiosity in the population of Polish older people inclusion of religiosity measures in mental health research is needed. The aim of the study was to investigate the relationship between religious involvement and mental health in older age.

Methods The sample population consisted of 350 randomly selected people aged 65 and over, living in Krakow. Religiosity was measured by assessing the following dimensions: ritualistic, experiential, ideological, consequential and intellectual. Religious coping and support were also measured. Health was assessed using SF-20, HADS, Will-to-live, DUKE Social Support and Loneliness Scales. Statistical analysis was done using K-means cluster analysis and multivariate regression analysis.

Results Two clusters were extracted in cluster analysis: cluster 1 (those who scored higher on each dimension of religiosity, ie, more religiously involved) and cluster 2 (less involved). Multivariate linear regression analysis showed that those more involved had better mental health $(\beta=0.182)$, were less depressed $(\beta=-0.236)$ and had more will to live $(\beta=0.186)$ in comparison with those less involved. Regression analysis for particular dimensions of religiosity showed that consequential and experiential dimension significantly influenced the level of depression ( $\beta=-0.202$ and -0.185 respectively); consequential dimension also influenced mental health $(\beta=0.228)$. Respondents who were engaged in positive religious coping responses had better mental health than those who didn't $(\beta=0.240)$. Emotional support provided, received and anticipated from the members of religious congregation decreased the level of social loneliness ( $\beta=-0.229,-0.238$ and -0.313 respectively).

Conclusions Religious involvement is a significant predictor of mental health in older age. 